\title{
Norm and Essential Norm of an Integral-Type Operator from the Dirichlet Space to the Bloch-Type Space on the Unit Ball
}

\author{
Stevo Stević \\ Mathematical Institute of the Serbian Academy of Sciences, Knez Mihailova 36/III, 11000 Beograd, Serbia \\ Correspondence should be addressed to Stevo Stević, sstevic@ptt.rs \\ Received 1 August 2010; Accepted 1 October 2010 \\ Academic Editor: Irena Lasiecka \\ Copyright (C) 2010 Stevo Stević. This is an open access article distributed under the Creative \\ Commons Attribution License, which permits unrestricted use, distribution, and reproduction in \\ any medium, provided the original work is properly cited. \\ Operator norm and essential norm of an integral-type operator, recently introduced by this author, \\ from the Dirichlet space to the Bloch-type space on the unit ball in $\mathbb{C}^{n}$ are calculated here.
}

\section{Introduction}

Let $\mathbb{B}^{n}=\mathbb{B}$ be the open unit ball in $\mathbb{C}^{n}, \mathbb{B}^{1}=\mathbb{D}$ the open unit disk in $\mathbb{C}, H(\mathbb{B})$ the class of all holomorphic functions on $\mathbb{B}$, and $H^{\infty}(\mathbb{B})$, the space consisting of all $f \in H(\mathbb{B})$ such that $\|f\|_{\infty}=\sup _{z \in \mathbb{B}}|f(z)|<\infty$.

For an $f \in H(\mathbb{B})$ with the Taylor expansion $f(z)=\sum_{\alpha} a_{\alpha} z^{\alpha}$, let

$$
\Re(z)=\sum_{\alpha}|\alpha| a_{\alpha} z^{\alpha}
$$

be the radial derivative of $f$, where $\alpha=\left(\alpha_{1}, \ldots, \alpha_{n}\right)$ is a multi-index, $|\alpha|=\alpha_{1}+\cdots+\alpha_{n}$ and $z^{\alpha}=z_{1}^{\alpha_{1}} \cdots z_{n}^{\alpha_{n}}$. Let $\alpha !=\alpha_{1} ! \cdots \alpha_{n} !$

The Dirichlet space $\Phi^{2}(\mathbb{B})=\Phi^{2}$ contains all $f(z)=\sum_{\alpha} a_{\alpha} z^{\alpha} \in H(\mathbb{B})$, such that

$$
\|f\|_{\Phi^{2}}^{2}:=|f(0)|^{2}+\sum_{\alpha}|\alpha| \frac{\alpha !}{|\alpha| !}\left|a_{\alpha}\right|^{2}<\infty
$$


The quantity $\|f\|_{\Phi^{2}}$ is a norm on $\Phi^{2}$ which for $n=1$ is equal to usual norm

$$
\|f\|_{\Phi^{2}(\mathbb{D})}=\left(|f(0)|^{2}+\int_{\mathbb{D}}\left|f^{\prime}(z)\right|^{2} d A(z)\right)^{1 / 2},
$$

where $d A(z)=(1 / \pi) r d r d \theta$ is the normalized area measure on $\mathbb{D}$.

The inner product, between two functions

$$
f(z)=\sum_{\alpha} a_{\alpha} z^{\alpha}, \quad g(z)=\sum_{\alpha} b_{\alpha} z^{\alpha},
$$

on $\Phi^{2}$ is defined by

$$
\langle f, g\rangle:=f(0) \overline{g(0)}+\sum_{\alpha}|\alpha| \frac{\alpha !}{|\alpha| !} a_{\alpha} \bar{b}_{\alpha}
$$

For $\alpha \neq 0$, let

$$
e_{\alpha}(z)=\sqrt{\frac{|\alpha| !}{|\alpha| \alpha !}} z^{\alpha}, \quad z \in \mathbb{B}
$$

and $e_{0}(z) \equiv 1$, then it is easy to see that the family $\left\{e_{\alpha}\right\}$ is an orthonormal basis for $\Phi^{2}$, and hence the reproducing kernel $K_{w}(z)$ for $\Phi^{2}$ is given by ([1]) as follows:

$$
K_{w}(z)=1+\sum_{\alpha \neq 0} \frac{|\alpha| !}{|\alpha| \alpha !} z^{\alpha} \bar{w}^{\alpha}=1+\ln \frac{1}{1-\langle z, w\rangle}
$$

where $\langle z, w\rangle=\sum_{j=1}^{n} z_{j} \bar{w}_{j}$ is the inner product in $\mathbb{C}^{n}$.

Clearly for each $f \in \Phi^{2}$ and $w \in \mathbb{B}$, the next reproducing formula holds:

$$
f(w)=\left\langle f, K_{w}\right\rangle
$$

Note that for $f=K_{w}$ from (1.8), we obtain

$$
K_{w}(w)=\left\|K_{w}\right\|_{\Phi^{2}}^{2}=\ln \frac{e}{1-|w|^{2}}
$$

Also, by the Cauchy-Schwarz inequality and (1.9), we have that, for each $f \in \Phi^{2}$ and $w \in \mathbb{B}$,

$$
|f(w)|=\left|\left\langle f, K_{w}\right\rangle\right| \leq\|f\|_{\Phi^{2}}\left\|K_{w}\right\|_{\Phi^{2}}=\|f\|_{\Phi^{2}}\left(\ln \frac{e}{1-|w|^{2}}\right)^{1 / 2} .
$$

Note that inequality (1.10) is exact since it is attained for $f=K_{w}$. 
The weighted-type space $H_{\mu}^{\infty}(\mathbb{B})=H_{\mu}^{\infty}([2,3])$ consists of all $f \in H(\mathbb{B})$ such that

$$
\|f\|_{H_{\mu}^{\infty}}:=\sup _{z \in \mathbb{B}} \mu(z)|f(z)|<\infty,
$$

where $\mu$ is a positive continuous function on $\mathbb{B}$ (weight).

The Bloch-type space $\mathbb{B}_{\mu}(\mathbb{B})=\mathbb{B}_{\mu}$ consists of all $f \in H(\mathbb{B})$ such that

$$
\|f\|_{\mathcal{B}_{\mu}}:=|f(0)|+\sup _{z \in \mathbb{B}} \mu(z)|\Re f(z)|<\infty,
$$

where $\mu$ is a weight.

Let $g \in H(\mathbb{D}), g(0)=0$, and $\varphi$ be a holomorphic self-map of $\mathbb{B}$, then the following integral-type operator:

$$
P_{\varphi}^{g}(f)(z)=\int_{0}^{1} f(\varphi(t z)) g(t z) \frac{d t}{t}, \quad z \in \mathbb{B}, f \in H(\mathbb{B})
$$

has been recently introduced in [4] and considerably studied (see, e.g., [5-8] and the related references therein). For some related operators, see also [9-16] and the references therein.

Usual problem in this research area is to provide function-theoretic characterizations for when $\varphi$ and $g$ induce bounded or compact integral-type operator on spaces of holomorphic functions. Majority of papers only find asymptotics of operator norm of linear operators. Somewhat concrete but perhaps more interesting problem is to calculate operator norm of these operators between spaces of holomorphic functions on various domains. Some results on this problem can be found, for example, in [3, 17-26] (for related results see also $[7,27-32])$. Having published paper [3], we started with systematic investigation of methods for calculating operator norms of concrete operators between spaces of holomorphic function.

Here, we calculate the operator norm as well as the essential norm of the operator $P_{\varphi}^{g}: \Phi^{2} \rightarrow \mathcal{B}_{\mu}$, considerably extending our recent result in note [33].

\section{Auxiliary Results}

In this section, we quote several auxiliary results which are used in the proofs of the main results.

Lemma 2.1 (see [4]). Let $g \in H(\mathbb{B}), g(0)=0$, and $\varphi$ be a holomorphic self-map of $\mathbb{B}$, then

$$
\mathfrak{R} P_{\varphi}^{g}(f)(z)=g(z) f(\varphi(z)) .
$$

The next Schwartz-type lemma ([34]) can be proved in a standard way. Hence, we omit its proof. 
Lemma 2.2. Assume that $g \in H(\mathbb{B}), g(0)=0, \mu$ is a weight, and $\varphi$ is an analytic self-map of $\mathbb{B}$, then $P_{\varphi}^{g}: \Phi^{2} \rightarrow \mathcal{B}_{\mu}$ is compact if and only if $P_{\varphi}^{g}: \Phi^{2} \rightarrow \mathcal{B}_{\mu}$ is bounded and for any bounded sequence $\left(f_{k}\right)_{k \in \mathbb{N}}$ in $\Phi^{2}$ converging to zero uniformly on compacts of $\mathbb{B}$ as $k \rightarrow \infty$, one has

$$
\lim _{k \rightarrow \infty}\left\|P_{\varphi}^{g}\left(f_{k}\right)\right\|_{\mathcal{B}_{\mu}}=0
$$

Lemma 2.3. Assume that $g \in H(\mathbb{B}), g(0)=0, \mu$ is a weight, $\varphi$ is an analytic self-map of $\mathbb{B}$ such that $\|\varphi\|_{\infty}<1$, and the operator $P_{\varphi}^{g}: \Phi^{2} \rightarrow \mathbb{B}_{\mu}$ is bounded, then $P_{\varphi}^{g}: \Phi^{2} \rightarrow \mathbb{B}_{\mu}$ is compact.

Proof. First note that since $P_{\varphi}^{g}: \boldsymbol{\Phi}^{2} \rightarrow \boldsymbol{B}_{\mu}$ is bounded and $f_{0}(z) \equiv 1 \in \boldsymbol{\Phi}^{2}$ by Lemma 2.1, it follows that $\mathfrak{R} P_{\varphi}^{g}\left(f_{0}\right)=g \in H_{\mu}^{\infty}$. Now, assume that $\left(f_{k}\right)_{k \in \mathbb{N}}$ is a bounded sequence in $\Phi^{2}$ converging to zero on compacts of $\mathbb{B}$ as $k \rightarrow \infty$, then we have

$$
\left\|P_{\varphi}^{g}\left(f_{k}\right)\right\|_{\mathcal{B}_{\mu}} \leq\|g\|_{H_{\mu}^{\infty}} \sup _{w \in \varphi(\mathbb{B})}\left|f_{k}(w)\right| \longrightarrow 0
$$

as $k \rightarrow \infty$, since $\varphi(\mathbb{B})$ is contained in the ball $|w| \leq\|\varphi\|_{\infty}$ which is a compact subset of $\mathbb{B}$, according to the assumption $\|\varphi\|_{\infty}<1$. Hence, by Lemma 2.2, the operator $P_{\varphi}^{g}: \mathscr{\Phi}^{2} \rightarrow \mathbb{B}_{\mu}$ is compact.

\section{Operator Norm of $P_{\varphi}^{g}: \mathscr{D}^{2} \rightarrow \boldsymbol{B}_{\mu}$}

In this section, we calculate the operator norm of $P_{\varphi}^{g}: \Phi^{2} \rightarrow \mathbb{B}_{\mu}$.

Theorem 3.1. Assume that $g \in H(\mathbb{B}), g(0)=0$, and $\varphi$ is a holomorphic self-map of $\mathbb{B}$, then

$$
\left\|P_{\varphi}^{g}\right\|_{\Phi^{2} \rightarrow B_{\mu}}=\sup _{z \in \mathbb{B}} \mu(z)|g(z)|\left(\ln \frac{e}{1-|\varphi(z)|^{2}}\right)^{1 / 2}=: L .
$$

Proof. Using Lemma 2.1, reproducing formula (1.8), the Cauchy-Schwarz inequality, and finally (1.9), we get that, for each $f \in \Phi^{2}$ and $w \in \mathbb{B}$,

$$
\begin{aligned}
\mu(w)\left|\mathfrak{R} P_{\varphi}^{g}(w)\right| & =\mu(w)|g(w)||f(\varphi(w))| \\
& =\mu(w)|g(w)|\left|\left\langle f, K_{\varphi(w)}\right\rangle\right| \\
& \leq \mu(w)|g(w)|\|f\|_{\Phi^{2}}\left\|K_{\varphi(w)}\right\|_{\Phi^{2}} \\
& =\|f\|_{\Phi^{2}} \mu(w)|g(w)|\left(\ln \frac{e}{1-|\varphi(w)|^{2}}\right)^{1 / 2} .
\end{aligned}
$$


Taking the supremum in (3.2) over $w \in \mathbb{B}$ as well as the supremum over the unit ball in $\Phi^{2}$ and using the fact $P_{\varphi}^{g}(f)(0)=0$, for each $f \in H(\mathbb{B})$, which follows from the assumption $g(0)=0$, we get

$$
\left\|P_{\varphi}^{g}\right\|_{\Phi^{2} \rightarrow B_{\mu}} \leq L
$$

Now assume that the operator $P_{\varphi}^{g}: \Phi^{2} \rightarrow \mathbb{B}_{\mu}$ is bounded. From (1.9), we obtain that, for each $w \in \mathbb{B}$,

$$
\begin{aligned}
\left(\ln \frac{e}{1-|\varphi(w)|^{2}}\right)^{1 / 2}\left\|P_{\varphi}^{g}\right\|_{\mathbb{\Phi}^{2} \rightarrow B_{\mu}} & =\left\|K_{\varphi(w)}\right\|_{\Phi^{2}}\left\|P_{\varphi}^{g}\right\|_{\mathbb{\Phi}^{2} \rightarrow B_{\mu}} \\
& \geq\left\|P_{\varphi}^{g}\left(K_{\varphi(w)}\right)\right\|_{\mathcal{B}_{\mu}} \\
& =\sup _{z \in \mathbb{B}} \mu(z)|g(z)|\left|K_{\varphi(w)}(\varphi(z))\right| \\
& \geq \mu(w)\left|g(w) \| K_{\varphi(w)}(\varphi(w))\right| .
\end{aligned}
$$

From (1.9) and (3.4), it follows that

$$
L \leq\left\|P_{\varphi}^{g}\right\|_{\Phi^{2} \rightarrow B_{\mu}} .
$$

Hence, if $P_{\varphi}^{g}: \Phi^{2} \rightarrow \mathcal{B}_{\mu}$ is bounded, then from (3.3) and (3.5) we obtain (3.1).

In the case $P_{\varphi}^{g}: \Phi^{2} \rightarrow \boldsymbol{B}_{\mu}$ is unbounded, the result follows from inequality (3.3).

\section{Essential Norm of $P_{\varphi}^{g}: \Phi^{2} \rightarrow B_{\mu}$}

Let $X$ and $Y$ be Banach spaces, and let $L: X \rightarrow Y$ be a bounded linear operator. The essential norm of the operator $L: X \rightarrow Y,\|L\|_{e, X \rightarrow Y}$, is defined as follows:

$$
\|L\|_{e, X \rightarrow Y}=\inf \left\{\|L+K\|_{X \rightarrow Y}: K \text { is compact from } X \text { to } Y\right\},
$$

where $\|\cdot\|_{X \rightarrow Y}$ denote the operator norm.

From this and since the set of all compact operators is a closed subset of the set of bounded operators, it follows that $L$ is compact if and only if

$$
\|L\|_{e, X \rightarrow Y}=0 .
$$

Here, we calculate the essential norm of the operator $P_{\varphi}^{g}: \Phi^{2} \rightarrow \mathcal{B}_{\mu}$. 
Theorem 4.1. Assume that $g \in H(\mathbb{B}), g(0)=0, \mu$ is a weight, $\varphi$ is a holomorphic self-map of $\mathbb{B}$, and $P_{\varphi}^{g}: \Phi^{2} \rightarrow \mathbb{B}_{\mu}$ is bounded. If $\|\varphi\|_{\infty}<1$, then $\left\|P_{\varphi}^{g}\right\|_{e, \Phi^{2} \rightarrow B_{\mu}}=0$, and if $\|\varphi\|_{\infty}=1$, then

$$
\left\|P_{\varphi}^{g}\right\|_{e, \mathbb{Q}^{2} \rightarrow B_{\mu}}=\limsup _{|\varphi(z)| \rightarrow 1} \mu(z)|g(z)|\left(\ln \frac{e}{1-|\varphi(z)|^{2}}\right)^{1 / 2} .
$$

Proof. Since $P_{\varphi}^{g}: \Phi^{2} \rightarrow \mathbb{B}_{\mu}$ is bounded, for the test function $f(z) \equiv 1$, we get $g \in H_{\mu}^{\infty}$. If $\|\varphi\|_{\infty}<1$, then from Lemma 2.3 it follows that $P_{\varphi}^{g}: \boldsymbol{\Phi}^{2} \rightarrow \boldsymbol{B}_{\mu}$ is compact which is equivalent with $\left\|P_{\varphi}^{g}\right\|_{e, \Phi^{2} \rightarrow B_{\mu}}=0$. On the other hand, it is clear that in this case the condition $|\varphi(z)| \rightarrow 1$ is vacuous, so that (4.3) is vacuously satisfied.

Now assume that $\|\varphi\|_{\infty}=1$, and that $\left(\varphi\left(z_{k}\right)\right)_{k \in \mathbb{N}}$ is a sequence in $\mathbb{B}$ such that $\left|\varphi\left(z_{k}\right)\right| \rightarrow$ 1 as $k \rightarrow \infty$. For $w \in \mathbb{B}$ fixed, set

$$
f_{w}(z)=\frac{\ln (e /(1-\langle z, w\rangle))}{\left(\ln \left(e /\left(1-|w|^{2}\right)\right)\right)^{1 / 2}}, \quad z \in \mathbb{B} .
$$

By (1.9), we have that $\left\|f_{w}\right\|_{\Phi^{2}}=1$, for each $w \in \mathbb{B}$. Hence, the sequence $\left(f_{\varphi\left(z_{k}\right)}\right)_{k \in \mathbb{N}}$ is such that $\left\|f_{\varphi\left(z_{k}\right)}\right\|_{\Phi^{2}}=1$, for each $k \in \mathbb{N}$, and clearly it converges to zero uniformly on compacts of $\mathbb{B}$. From this and by [35, Theorems 6.19], it easily follows that $f_{\varphi\left(z_{k}\right)} \rightarrow 0$ weakly in $\Phi^{2}$, as $k \rightarrow \infty$. Hence, for every compact operator $K: \Phi^{2} \rightarrow \mathbb{B}_{\mu}$, we have that

$$
\lim _{k \rightarrow \infty}\left\|K f_{\varphi\left(z_{k}\right)}\right\|_{\mathcal{B}_{\mu}}=0
$$

Thus, for every such sequence and for every compact operator $K: \Phi^{2} \rightarrow \mathcal{B}_{\mu}$, we have that

$$
\begin{aligned}
\left\|P_{\varphi}^{g}+K\right\|_{\Phi^{2} \rightarrow B_{\mu}} & \geq \limsup _{k \rightarrow \infty} \frac{\left\|P_{\varphi}^{g} f_{\varphi\left(z_{k}\right)}\right\|_{\mathcal{B}_{\mu}}-\left\|K f_{\varphi\left(z_{k}\right)}\right\|_{\mathcal{B}_{\mu}}}{\left\|f_{\varphi\left(z_{k}\right)}\right\|_{\Phi^{2}}} \\
& =\limsup _{k \rightarrow \infty}\left\|P_{\varphi}^{g} f_{\varphi\left(z_{k}\right)}\right\|_{\mathcal{B}_{\mu}} \\
& \geq \limsup _{k \rightarrow \infty} \mu\left(z_{k}\right)\left|g\left(z_{k}\right) f_{\varphi\left(z_{k}\right)}\left(\varphi\left(z_{k}\right)\right)\right| \\
& =\limsup _{n \rightarrow \infty} \mu\left(z_{k}\right)\left|g\left(z_{k}\right)\right|\left(\ln \frac{e}{1-\left|\varphi\left(z_{k}\right)\right|^{2}}\right)^{1 / 2} .
\end{aligned}
$$
obtain

Taking the infimum in (4.6) over the set of all compact operators $K: \Phi^{2} \rightarrow \mathcal{B}_{\mu}$, we

$$
\left\|P_{\varphi}^{g}\right\|_{e, \mathbb{Q}^{2} \rightarrow B_{\mu}} \geq \limsup _{n \rightarrow \infty} \mu\left(z_{k}\right)\left|g\left(z_{k}\right)\right|\left(\ln \frac{e}{1-\left|\varphi\left(z_{k}\right)\right|^{2}}\right)^{1 / 2}
$$

from which an inequality in (4.3) follows. 
In the sequel, we prove the reverse inequality. Assume that $\left(r_{l}\right)_{l \in \mathbb{N}}$ is a sequence of positive numbers which increasingly converges to 1 . Consider the operators defined by

$$
\left(P_{r_{l} \varphi}^{g} f\right)(z)=\int_{0}^{1} f\left(r_{l} \varphi(t z)\right) g(t z) \frac{d t}{t}, \quad l \in \mathbb{N}
$$

Since $\left\|r_{l} \varphi\right\|_{\infty}<1$, by Lemma 2.3, we have that these operators are compact.

Since $P_{\varphi}^{g}: \Phi^{2} \rightarrow B_{\mu}$ is bounded, then $g \in H_{\mu}^{\infty}$. Let $\rho \in(0,1)$ be fixed for a moment. By Lemma 2.1, we get

$$
\begin{aligned}
& \left\|P_{\varphi}^{g}-P_{r_{l} \varphi}^{g}\right\|_{\Phi^{2} \rightarrow B_{\mu}}=\sup _{\|f\|_{\mathbb{Q}^{2}} \leq 1} \sup _{z \in \mathbb{B}} \mu(z)|g(z)|\left|f(\varphi(z))-f\left(r_{l} \varphi(z)\right)\right| \\
& \leq \sup _{\|f\|_{\mathbb{Q}^{2} \leq 1} \leq 1|\varphi(z)| \leq \rho} \sup _{\mid} \mu(z)|g(z)|\left|f(\varphi(z))-f\left(r_{l} \varphi(z)\right)\right| \\
& +\sup _{\|f\|_{\otimes^{2}} \leq 1|\varphi(z)|>\rho} \sup _{\mid>\rho} \mu(z)|g(z)|\left|f(\varphi(z))-f\left(r_{l} \varphi(z)\right)\right| \\
& \leq\|g\|_{H_{\mu}^{\infty}} \sup _{\|f\|_{\Phi^{2} \leq 1}} \sup _{|\varphi(z)| \leq \rho}\left|f(\varphi(z))-f\left(r_{l} \varphi(z)\right)\right| \\
& +\sup _{\|f\|_{\mathbb{Q}^{2}} \leq 1|\varphi(z)|>\rho} \sup _{\mid} \mu(z)|g(z)|\left|f(\varphi(z))-f\left(r_{l} \varphi(z)\right)\right| .
\end{aligned}
$$

Further, we have

$$
\left\|f-f_{r}\right\|_{\Phi^{2}}^{2}=\sum_{\alpha}|\alpha| \frac{\alpha !}{|\alpha| !}\left|a_{\alpha}\right|^{2}\left(1-r^{|\alpha|}\right)^{2} \leq \sum_{\alpha}|\alpha| \frac{\alpha !}{|\alpha| !}\left|a_{\alpha}\right|^{2} \leq\|f\|_{\Phi^{2}}
$$

From (1.10), (4.11), and the fact $f(z)-f(r z) \in \Phi^{2}$, we obtain

$$
|f(z)-f(r z)| \leq\|f\|_{\Phi^{2}}\left(\ln \frac{e}{1-|z|^{2}}\right)^{1 / 2}
$$

in particular

$$
\left|f(\varphi(z))-f\left(r_{l} \varphi(z)\right)\right| \leq\|f\|_{\Phi^{2}}\left(\ln \frac{e}{1-|\varphi(z)|^{2}}\right)^{1 / 2} .
$$

Let

$$
I_{l}:=\sup _{\|f\|_{\Phi^{2}} \leq 1|\varphi(z)| \leq \rho} \sup _{\left|f(\varphi(z))-f\left(r_{l} \varphi(z)\right)\right|} \mid f
$$


The mean value theorem along with the subharmonicity of the moduli of partial derivatives of $f$, well-known estimates among the partial derivatives of analytic functions, Theorem 6.2, and Proposition 6.2 in [35], yield

$$
\begin{aligned}
& I_{l} \leq \sup _{\|f\|_{\Phi^{2}} \leq 1} \sup _{|\varphi(z)| \leq \rho}\left(1-r_{l}\right)|\varphi(z)| \sup _{|w| \leq \rho}|\nabla f(w)| \\
& \leq C_{\rho}\left(1-r_{l}\right) \sup _{\|f\|_{\Phi^{2} \leq 1}}\left(\sum_{j=1}^{[n / p]}\left|\nabla^{j} f(0)\right|+\sup _{|w| \leq(1+\rho) / 2}\left|\nabla^{[n / p]+1} f(w)\right|\right) \\
& \leq C_{\rho}\left(1-r_{l}\right) \sup _{\|f\|_{\Phi^{2}} \leq 1}\left(\sum_{j=1}^{[n / p]}\left|\nabla^{j} f(0)\right|\right. \\
& \left.\qquad\left(\int_{|w| \leq(3+\rho) / 4}\left|\nabla^{[n / p]+1} f(w)\right|^{2}\left(1-|w|^{2}\right)^{2([n / p]+1)} d \tau(w)\right)^{1 / 2}\right) \\
& \leq C_{\rho}\left(1-r_{l}\right) \sup _{\|f\|_{\Phi^{2}} \leq 1}\left(\sum_{j=1}^{[n / p]}\left|\nabla^{j} f(0)\right|+\int_{\mathbb{B}}\left|\nabla^{[n / p]+1} f(w)\right|^{2}\left(1-|w|^{2}\right)^{2([n / p]+1)} d \tau(w)\right)^{1 / 2} \\
& \leq C_{\rho}\left(1-r_{l}\right) \longrightarrow 0, \quad \text { as } l \longrightarrow \infty,
\end{aligned}
$$

where $d \tau(z)=d V(z) /\left(1-|z|^{2}\right)^{n+1}$ and $d V(z)$ is the Lebesgue volume measure on $\mathbb{B}$.

Using (4.13) in (4.10), letting $l \rightarrow \infty$ in (4.9), using (4.15), and then letting $\rho \rightarrow 1$, the reverse inequality follows, finishing the proof of the theorem.

\section{References}

[1] K. Zhu, "Distances and Banach spaces of holomorphic functions on complex domains," Journal of the London Mathematical Society, vol. 49, no. 1, pp. 163-182, 1994.

[2] K. D. Bierstedt and W. H. Summers, "Biduals of weighted Banach spaces of analytic functions," Journal of Australian Mathematical Society A, vol. 54, no. 1, pp. 70-79, 1993.

[3] S. Stević, "Norm of weighted composition operators from Bloch space to $H_{\mu}^{\infty}$ on the unit ball," Ars Combinatoria, vol. 88, pp. 125-127, 2008.

[4] S. Stević, "On a new operator from $H^{\infty}$ to the Bloch-type space on the unit ball," Utilitas Mathematica, vol. 77, pp. 257-263, 2008.

[5] S. G. Krantz and S. Stević, "On the iterated logarithmic Bloch space on the unit ball," Nonlinear Analysis. Theory, Methods E Applications, vol. 71, no. 5-6, pp. 1772-1795, 2009.

[6] S. Stević, "On a new operator from the logarithmic Bloch space to the Bloch-type space on the unit ball," Applied Mathematics and Computation, vol. 206, no. 1, pp. 313-320, 2008.

[7] S. Stević, "On a new integral-type operator from the Bloch space to Bloch-type spaces on the unit ball," Journal of Mathematical Analysis and Applications, vol. 354, no. 2, pp. 426-434, 2009.

[8] W. Yang, "On an integral-type operator between Bloch-type spaces," Applied Mathematics and Computation, vol. 215, no. 3, pp. 954-960, 2009.

[9] D. Gu, "Extended Cesàro operators from logarithmic-type spaces to Bloch-type spaces," Abstract and Applied Analysis, vol. 2009, Article ID 246521, 9 pages, 2009.

[10] Z. Hu, "Extended Cesàro operators on Bergman spaces," Journal of Mathematical Analysis and Applications, vol. 296, no. 2, pp. 435-454, 2004. 
[11] S. Stević, "Generalized composition operators from logarithmic Bloch spaces to mixed-norm spaces," Utilitas Mathematica, vol. 77, pp. 167-172, 2008.

[12] S. Stević, "Boundedness and compactness of an integral operator between $H^{\infty}$ and a mixed norm space on the polydisk," Siberian Mathematical Journal, vol. 50, no. 3, pp. 495-497, 2009.

[13] S. Stević, "Integral-type operators from a mixed norm space to a Bloch-type space on the unit ball," Siberian Mathematical Journal, vol. 50, no. 6, pp. 1098-1105, 2009.

[14] S. Stević, "Products of integral-type operators and composition operators from the mixed norm space to Bloch-type spaces," Siberian Mathematical Journal, vol. 50, no. 4, pp. 726-736, 2009.

[15] S. Stević, "On an integral operator between Bloch-type spaces on the unit ball," Bulletin des Sciences Mathematiques, vol. 134, no. 4, pp. 329-339, 2010.

[16] S. Stević, "Compactness of the Hardy-Littlewood operator on some spaces of harmonic functions," Siberian Mathematical Journal, vol. 50, no. 1, pp. 167-180, 2009.

[17] R. F. Allen and F. Colonna, "Weighted composition operators from $H^{\infty}$ to the Bloch space of a bounded homogeneous domain," Integral Equations and Operator Theory, vol. 66, no. 1, pp. 21-40, 2010.

[18] R. F. Allen and F. Collona, "Weighted composition operators on the Bloch space of a bounded homogeneous domain," Operator Theory: Advances and Applications, vol. 202, pp. 11-37, 2010.

[19] M. H. Shaabani and B. K. Robati, "On the norm of certain weighted composition operators on the Hardy space," Abstract and Applied Analysis, vol. 2009, Article ID 720217, 13 pages, 2009.

[20] S. Stević, "Norms of some operators from Bergman spaces to weighted and Bloch-type spaces," Utilitas Mathematica, vol. 76, pp. 59-64, 2008.

[21] S. Stević, "Norm and essential norm of composition followed by differentiation from $\alpha$-Bloch spaces to $H_{\mu}^{\infty}, "$ Applied Mathematics and Computation, vol. 207, no. 1, pp. 225-229, 2009.

[22] S. Stević, "Norm of weighted composition operators from $\alpha$-Bloch spaces to weighted-type spaces," Applied Mathematics and Computation, vol. 215, no. 2, pp. 818-820, 2009.

[23] S. Stević, "Weighted composition operators between Fock-type spaces in $\mathbb{C}^{N}$," Applied Mathematics and Computation, vol. 215, no. 7, pp. 2750-2760, 2009.

[24] S. Stević, "Weighted composition operators from weighted Bergman spaces to weighted-type spaces on the unit ball," Applied Mathematics and Computation, vol. 212, no. 2, pp. 499-504, 2009.

[25] S. Stević, "Norms of some operators on bounded symmetric domains," Applied Mathematics and Computation, vol. 216, no. 1, pp. 187-191, 2010.

[26] S.-I. Ueki, "Weighted composition operators on some function spaces of entire functions," Bulletin of the Belgian Mathematical Society. Simon Stevin, vol. 17, no. 2, pp. 343-353, 2010.

[27] S. Stević, "Essential norms of weighted composition operators from the $\alpha$-Bloch space to a weightedtype space on the unit ball," Abstract and Applied Analysis, vol. 2008, Article ID 279691, 11 pages, 2008.

[28] S. Stević, "Composition operators from the Hardy space to the Zygmund-type space on the upper half-plane," Abstract and Applied Analysis, vol. 2009, Article ID 161528, 8 pages, 2009.

[29] S. Stević, "Essential norm of an operator from the weighted Hilbert-Bergman space to the Bloch-type space," Ars Combinatoria, vol. 91, pp. 123-127, 2009.

[30] S. Stević, "Norms of some operators on the Bergman and the Hardy space in the unit polydisk and the unit ball," Applied Mathematics and Computation, vol. 215, no. 6, pp. 2199-2205, 2009.

[31] S.-I. Ueki, "Hilbert-Schmidt weighted composition operator on the Fock space," International Journal of Mathematical Analysis, vol. 1, no. 13-16, pp. 769-774, 2007.

[32] S.-I. Ueki, "Weighted composition operators on the Bargmann-Fock space," International Journal of Modern Mathematics, vol. 3, no. 3, pp. 231-243, 2008.

[33] S. Stević, "Norm of an integral-type operator from Dirichlet to Bloch space on the unit disk," Utilitas Mathematica, vol. 83, pp. 301-303, 2010.

[34] H. J. Schwartz, Composition operators on $H^{p}$, Thesis, University of Toledo, 1969.

[35] Kehe Zhu, Spaces of Holomorphic Functions in the Unit Ball, vol. 226, Springer, New York, NY, USA, 2005. 


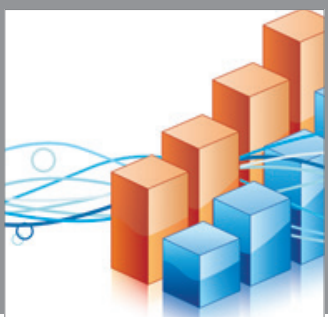

Advances in

Operations Research

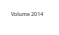

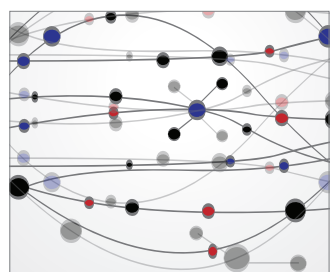

\section{The Scientific} World Journal
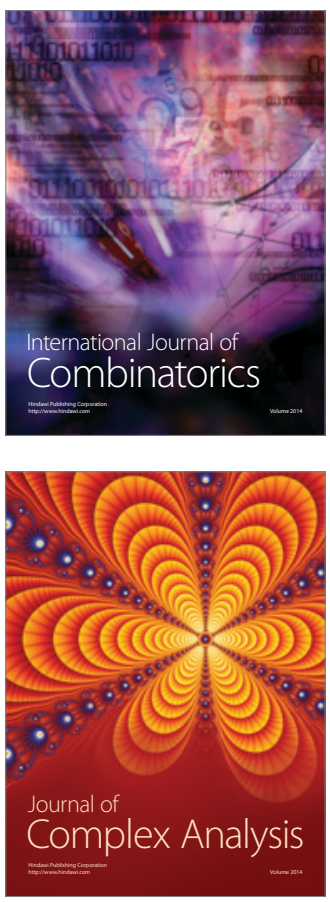

International Journal of

Mathematics and

Mathematical

Sciences
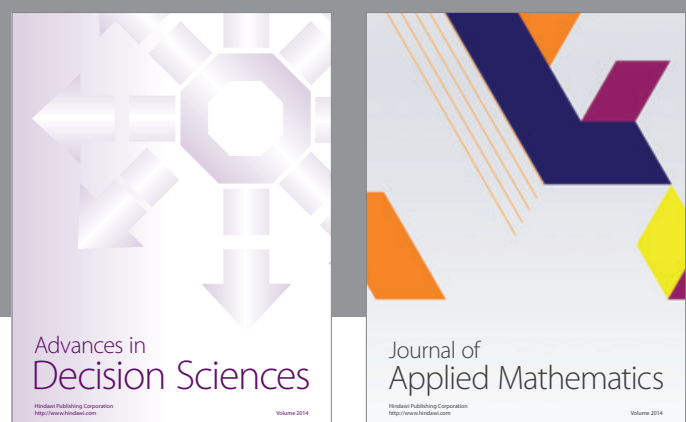

Journal of

Applied Mathematics
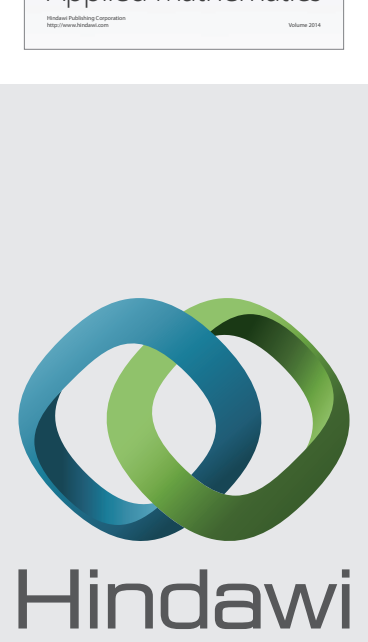

Submit your manuscripts at http://www.hindawi.com
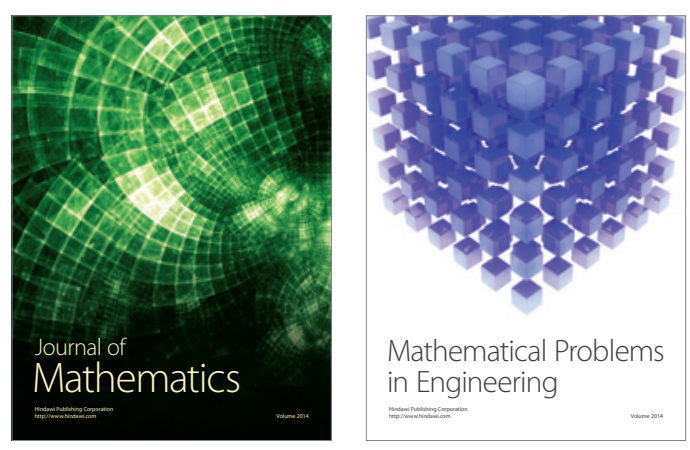

Mathematical Problems in Engineering
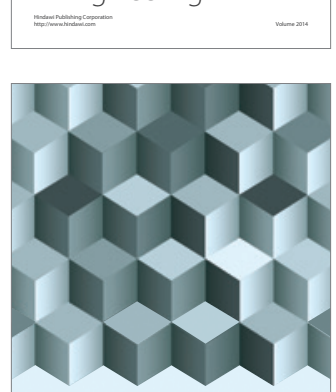

Journal of

Function Spaces
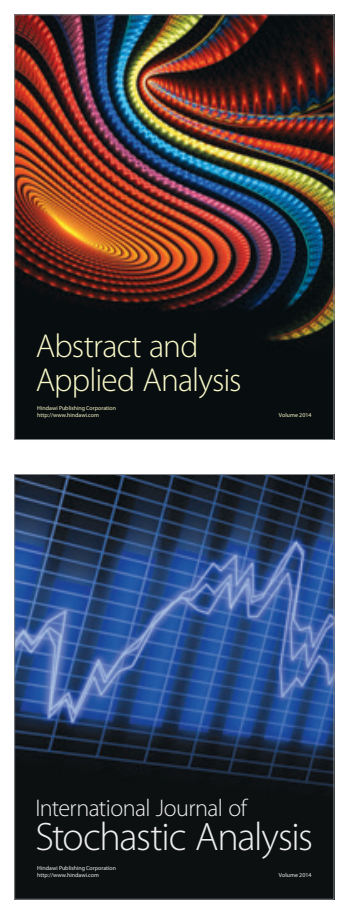

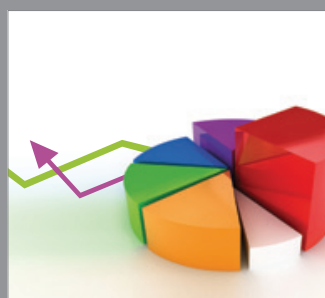

ournal of

Probability and Statistics

Promensencen
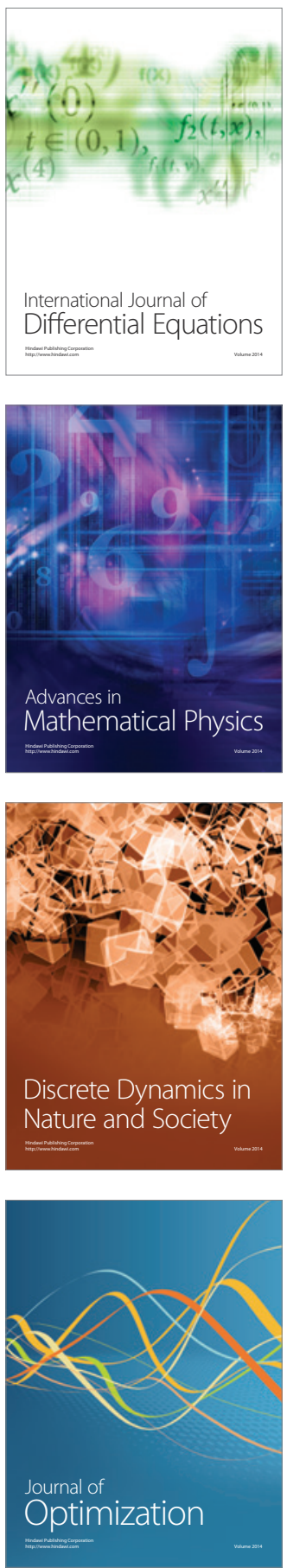